\title{
Fetal tracheolaryngeal airway obstruction: prenatal evaluation by sonography and MRI
}

\author{
Jesse Courtier • Liina Poder • Zhen J. Wang • \\ Antonio C. Westphalen • Benjamin M. Yeh • \\ Fergus V. Coakley
}

Received: 25 March 2010 /Revised: 14 July 2010 / Accepted: 27 July 2010 /Published online: 25 August 2010

(C) The Author(s) 2010. This article is published with open access at Springerlink.com

\begin{abstract}
We reviewed the sonographic and MRI findings of tracheolaryngeal obstruction in the fetus. Conditions that can cause tracheolaryngeal obstruction include extrinsic causes such as lymphatic malformation, cervical teratoma and vascular rings and intrinsic causes such as congenital high airway obstruction syndrome (CHAOS). Accurate distinction of these conditions by sonography or MRI can help facilitate parental counseling and management, including the decision to utilize the ex utero intrapartum treatment (EXIT) procedure.
\end{abstract}

Keywords Fetal tracheolaryngeal - Airway obstruction . Sonography $\cdot$ MRI

\section{Introduction}

Fetal tracheolaryngeal obstruction is a life-threatening condition with substantial morbidity and mortality. If unrecognized before delivery, the reported mortality is $80 \%$ to $100 \%$ [1]. Lifelong morbidity can result from emergency resuscitation accompanied by hypoxia, acidosis and anoxic brain injury. Causes of fetal tracheolaryngeal obstruction can be classified as extrinsic or intrinsic [2] Extrinsic obstruction might be caused by adjacent tumors

J. Courtier $\cdot$ L. Poder $\cdot$ Z. J. Wang $\cdot$ A. C. Westphalen $\cdot$

B. M. Yeh $\cdot$ F. V. Coakley $(\square)$

Department of Radiology, University of California San Francisco, Box 0628, M-372, 505 Parnassus Ave.,

San Francisco, CA 94143-0628, USA

e-mail: Fergus.Coakley@radiology.ucsf.edu such as lymphatic malformation, cervical teratoma or compression by a vascular ring. Intrinsic obstruction can also occur, leading to the constellation of findings known as congenital high airway obstruction syndrome (CHAOS). Prenatal imaging recognition and evaluation of fetal tracheolaryngeal obstruction has become increasingly important because the recently described ex utero intrapartum treatment (EXIT) procedure can be used to deliver affected fetuses by cesarean section under deep maternal and fetal anesthesia, allowing for safe airway control and management and greatly improving outcome. MRI is a useful means of imaging the anatomy of the fetal airway [3] (Fig. 1). Accordingly, the purpose of this pictorial essay is to review the sonographic and MRI findings of tracheolaryngeal obstruction in the fetus by describing the findings in intrinsic and extrinsic causes of obstruction and reviewing the associated management options.

\section{Intrinsic tracheolaryngeal obstruction}

Congenital high airway obstruction syndrome (CHAOS) is a rare form of intrinsic obstruction of the larynx or trachea that results in retention of bronchial secretions and subsequent pulmonary distention from the retained fluid [4]. Obstruction of the airway in CHAOS might be related to intrinsic atresia or stenosis of the larynx or upper trachea [4]. This obstruction leads to the constellation of pulmonary and extra-pulmonary findings. Pulmonary findings include dilated airways below the level of obstruction, hyperexpanded lungs and flattened diaphragms. Extra- 


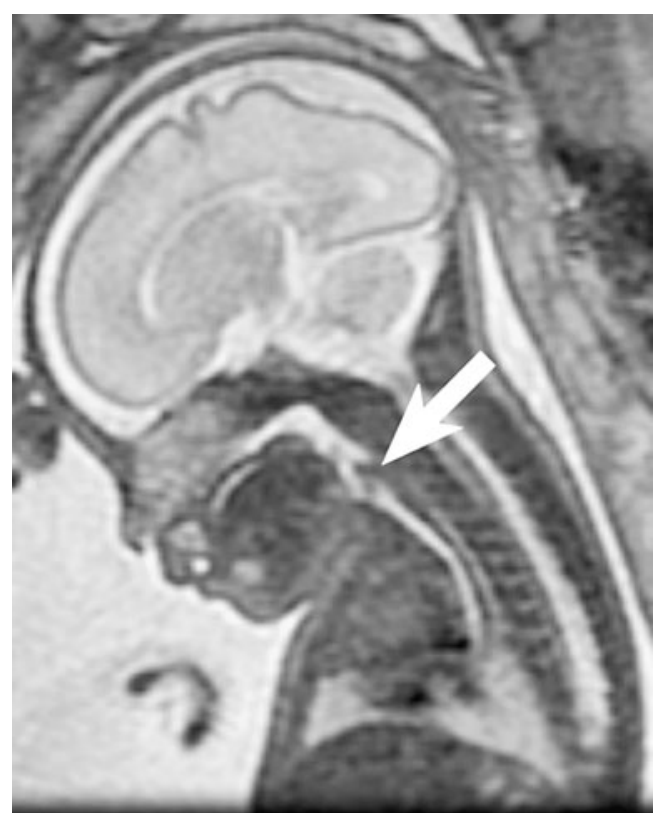

Fig. 1 Sagittal single-shot fast-spin echo (SSFSE) T2-W image depicts a normal upper airway in a fetus at 29 weeks' gestation. The aryepiglottic folds are well visualized (white arrow)

pulmonary findings include fetal hydrops or ascites and placentomegaly. Although the exact etiology is not clear, it is believed that hyperexpansion of the lungs results in flattening or eversion of the diaphragm. This abnormal hyperexpansion of the lungs is believed to cause impaired venous return to the heart. It has been proposed that this impaired venous return leads to the subsequent development of fetal hydrops and ascites [4, 5].

Sonographic findings of CHAOS have been well described [4-6], and these findings can also be recognized on MRI [5] (Figs. 2 and 3). MRI nicely depicts the findings that constitute CHAOS such as dilated airways below the level of obstruction, hyperexpanded and T2 hyperintense lungs, flattened or everted diaphragm, and fetal ascites [5]. Furthermore, MRI can help to better localize the level of obstruction [7-9]. Correct localization helps guide the decision between fetal versus neonatal intervention with the EXIT procedure [7, 9]. There is also potential prognostic indication for the success of the EXIT procedure, as obstruction low in the thoracic trachea has been described with failed EXIT procedure [7].

\section{Extrinsic tracheolaryngeal obstruction}

\section{Cervical teratoma}

The head and neck is the second commonest site of teratoma in the fetus [1]. These masses typically consist of both cystic and solid components and originate from the palate, nasopharynx or thyrocervical area (Fig. 4). If
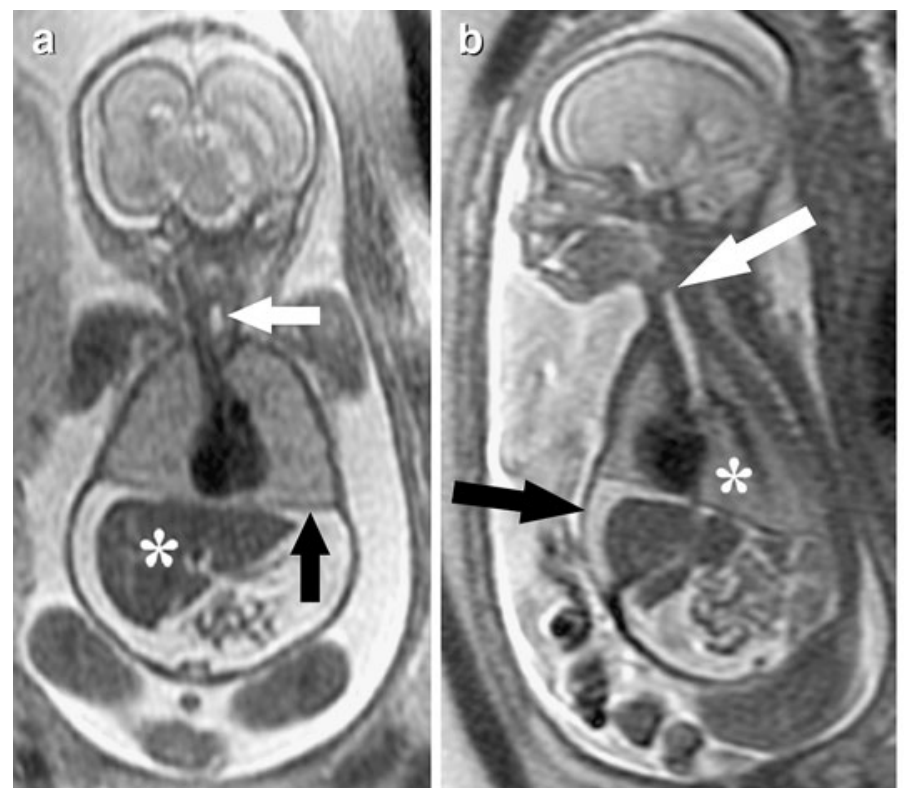

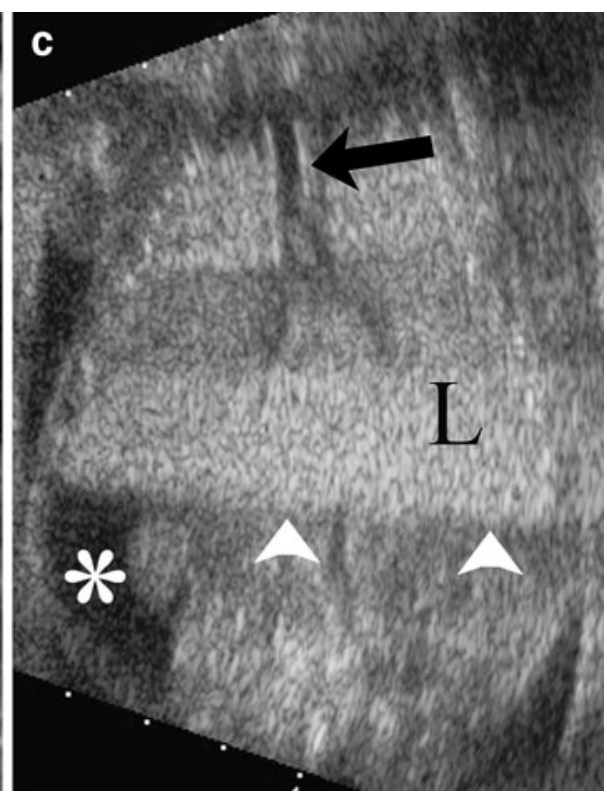

are prominent and fluid-filled. The constellation of findings is highly suggestive of CHAOS. c Coronal gray-scale sonographic images demonstrate echogenic lungs $(L)$. Flattening of the diaphragm is also appreciated (white arrowheads). Intraperitoneal ascites (asterisk) is also present. An EXIT procedure was performed with successful fetal tracheostomy. Postnatal laryngoscopy demonstrated laryngeal atresia secondary to underlying fusion of the false vocal cords
Fig. 2 MRI findings of CHAOS. a Coronal SSFSE T2-W image obtained in a 24-week 6-day fetus with suspected laryngeal atresia on prior level II US demonstrates expanded homogeneous lungs with flattened diaphragm (black arrow). The liver (asterisk) is surrounded by ascites. The trachea (white arrow) is also visible. b Sagittal SSFSE T2-W image of the same fetus demonstrates expanded lungs (asterisk) with intraperitoneal ascites (black arrow). The trachea (white arrow) and proximal mainstem bronchi 
Fig. 3 Coronal SSFSE T2-W image in a fetus at 25 weeks 1 day gestational age with polyhydramnios and fetal hydrops on US. This exam demonstrates a focal area of obstruction at the level of the larynx (white arrowhead) with dilation of the airways below the level of the obstruction (white arrow). There is flattening of the diaphragm (black arrow). Fetal ascites (asterisk) is also visible. Fetal bronchoscopy was performed at 27 weeks where a laryngeal web was discovered and lysed. EXIT procedure was performed at 35 weeks 5 days with bronchoscopy and tracheotomy

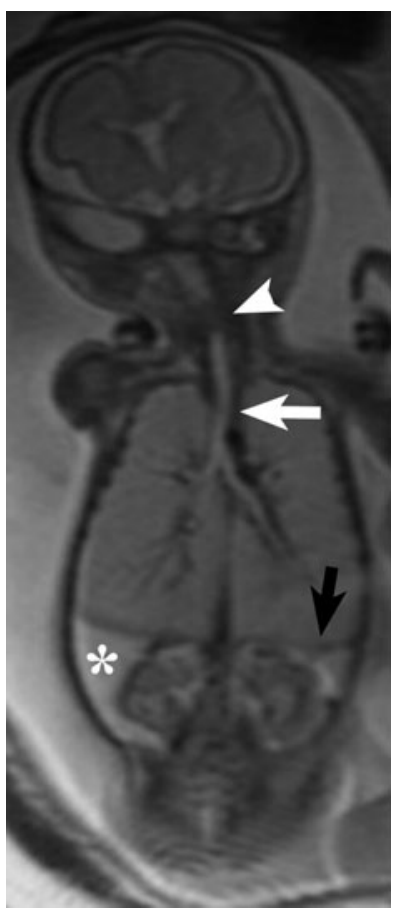

within the differential diagnosis include goiter, hemangioma, neuroblastoma and soft-tissue tumors [1].

Vascular anomalies

Extrinsic compression from vascular rings including double aortic arch has been described as resulting in significant airway obstruction leading to features mimicking CHAOS [2] (Fig. 7). Double aortic arch is a relatively common congenital anomaly of the aortic arch system resulting from failed regression of the fourth pharyngeal arch arteries. Postnatal symptoms develop because of compression of the trachea and esophagus, which are encircled by the left and right aortic arches. This entity classically manifests as noisy breathing during the first few weeks of life. Critical review of imaging findings in apparent CHAOS cases is necessary on prenatal sonography. Atypical features should lead to further imaging with fetal MRI or fetal echocardiography, which can help to identify and characterize extrinsic vascular anomalies. Recognition that double aortic arch or other cause of vascular rings including right aortic arch with aberrant left subclavian artery can mimic CHAOS on prenatal sonography might prevent misdiagnosis and facilitate appropriate treatment [2].

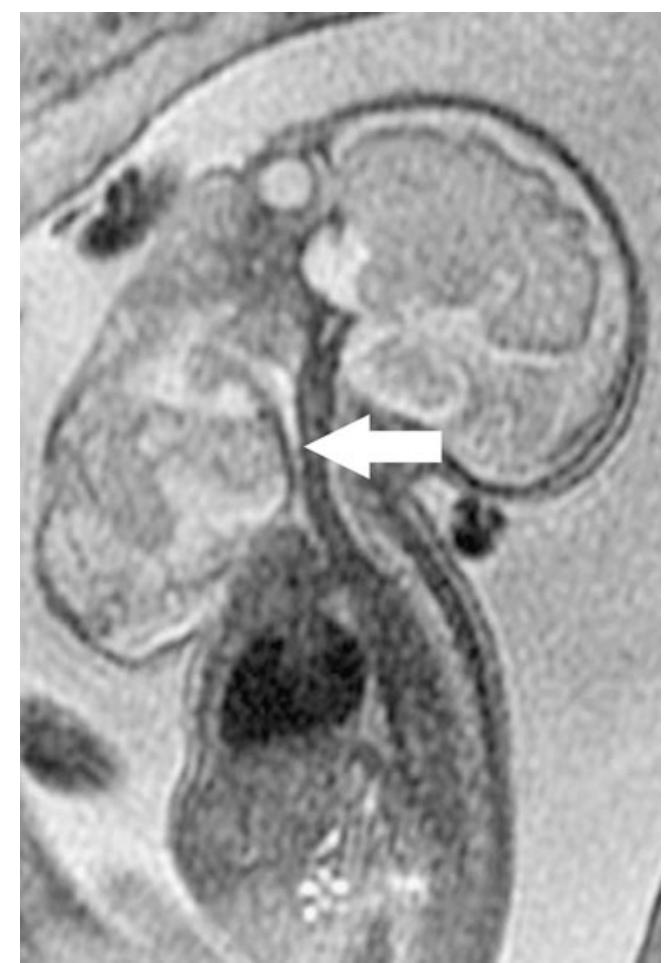

Fig. 4 Sagittal SSFSE T2-W image in a 30-week fetus with complex neck mass on level II US demonstrates a large cystic and solid mass arising from the anterior neck and upper chest extending superiorly to the right face and right orbit. There is severe stenosis of the subglottic region of the larynx (arrow). An EXIT procedure was subsequently performed on this patient and due to its extensive involvement, the mass was resected at the time of the procedure in order to establish an adequate airway 


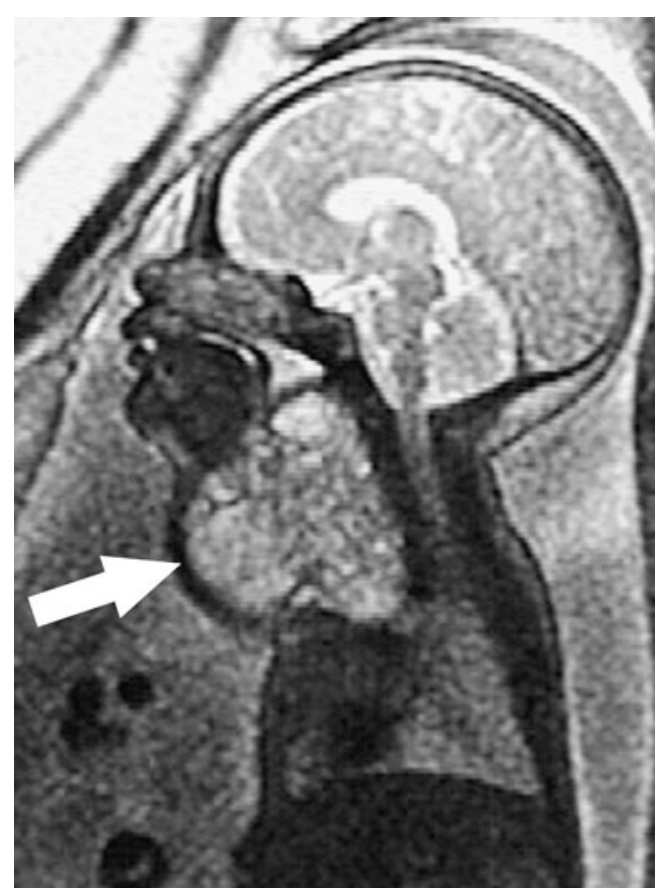

Fig. 5 Sagittal SSFSE T2-W image demonstrates a multiseptated T2 hyperintense mass in the anterior neck (arrow) resulting in mass effect on the subglottic upper airway. Although not hyperexpanded, the lungs do demonstrate hyperintense T2-W signal. This was found to be a lymphatic malformation following resection

\section{Management}

With the use of the ex utero intrapartum treatment (EXIT) procedure, a marked improvement in survival can now be accomplished. The EXIT procedure involves partial delivery of the fetus via a hysterotomy while both the placenta and umbilical cord remain intact. Halogenated agents are administered to the mother in order to promote uterine relaxation [4]. The fetus remains hemodynamically stable, as the uteroplacental gas exchange is maintained. Mean times of hemodynamic stability have ranged from 18 to $45 \mathrm{~min}$ [1]. This allows for a controlled environment to manage the airway, as opposed to a more emergent attempt at airway access at birth [1]. A common strategy is to first attempt intubation via direct laryngoscopy. If the airway is not adequately visualized then rigid bronchoscopy is attempted. If an endotracheal tube is unable to be passed, tracheotomy is the final option [4]. Although the EXIT procedure was initially developed to deliver fetuses with congenital diaphragmatic hernia after tracheal occlusion [4], it has also demonstrated success in the treatment of fetuses with obstructing neck masses and cleft lip and palate $[1,10-12]$.

In a study by Wagner and Harrison [11], an airway was established in $79 \%$ of the 29 cases reviewed using the EXIT procedure, with an overall survival rate of $69 \%$. Another study with 13 patients treated with the EXIT procedure for obstructive neck masses resulted in only one death [1]. Successful management of CHAOS has been achieved with a combination of fetal tracheostomy and delivery using the EXIT procedure [3, 11]. Fetal MRI has demonstrated its value in the facilitation of treatment planning in fetal upper airway obstruction. With a large field of view and high soft-tissue contrast, MRI can
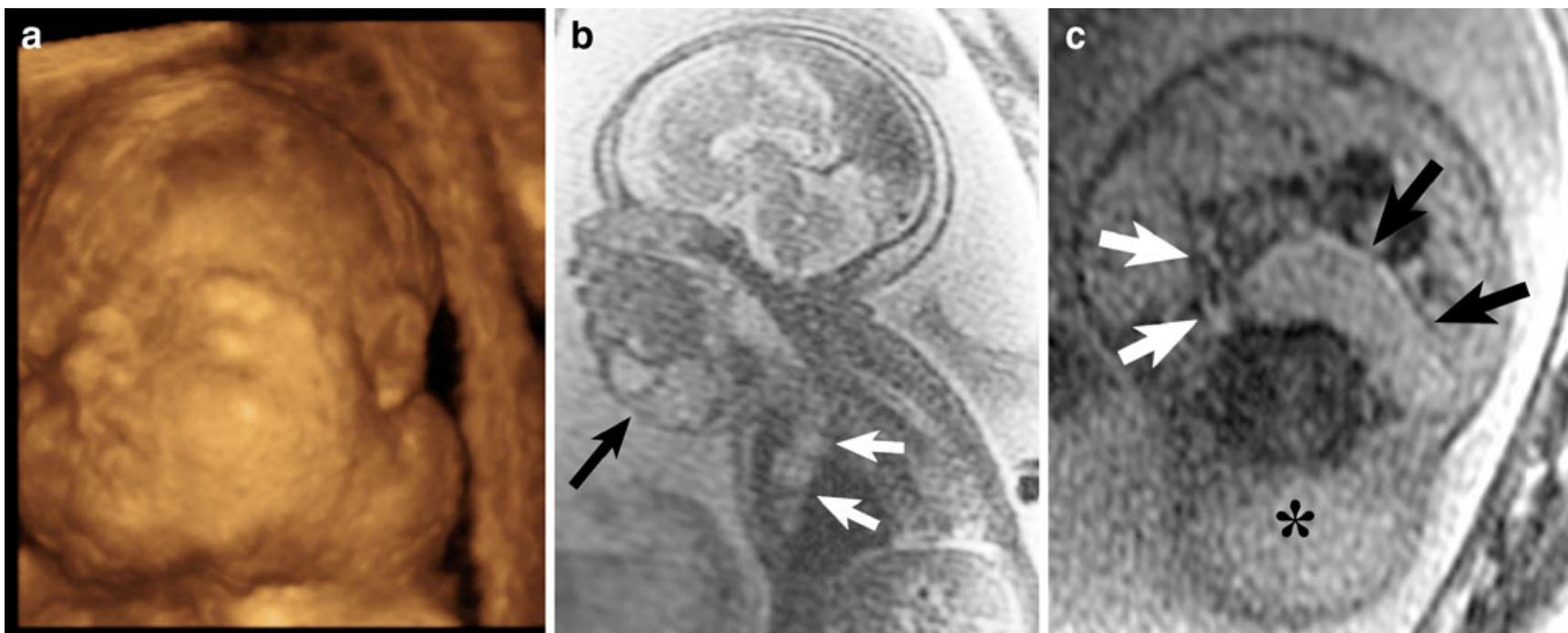

Fig. 6 Lymphatic malformation. a 3-D sonographic image in a 25-week fetus with suspected neck mass on prior US at an outside facility demonstrates a mass arising from the left lateral aspect of the fetal neck. b Sagittal SSFSE T2-W image in the same patient as Fig. 7 obtained at 31 weeks demonstrates a multiseptated T2 hyperintense mass in the anterior neck (black arrow) with extension into the anterior mediasti- num (white arrows), most consistent with lymphatic malformation. Secondary signs of airway obstruction were not present. c Axial SSFSE $\mathrm{T} 2-\mathrm{W}$ image demonstrates a cystic retropharyngeal component of the multiseptated mass (black arrows) that displaces the oropharynx (white arrows) anterolaterally. Hydrops of the soft tissues posterior to the cervical spine (asterisk) is also seen. This baby died shortly after birth 
Fig. 7 Images in a 26-year-old woman referred for suspected prenatal CHAOS. a Sagittal US image demonstrates expanded, echogenic lungs (asterisk) and a flattened hemidiaphragm. Fetal ascites is also visible (arrow). b Sagittal SSFSE T2-W MRI at 24 weeks' gestation demonstrates an expanded left lung and resultant flattening of the hemidiaphragm (arrowheads). Intraperitoneal ascites is visible $(A)$. Mild dilation of the left lower lobe bronchus (arrow) is also visible. c Coronal SSFSE T2-W MRI demonstrates narrowing of the intrathoracic trachea between the dominant right aortic arch (black arrow) and the smaller left aortic arch (white arrow). White asterisk demarcates the right lung. d Axial image from fetal echocardiogram demonstrates left aortic arch $(L A A)$ and right aortic arch (RAA), confirming the presence of a double aortic arch resulting in upper airway obstruction (Images reprinted with permission [2])
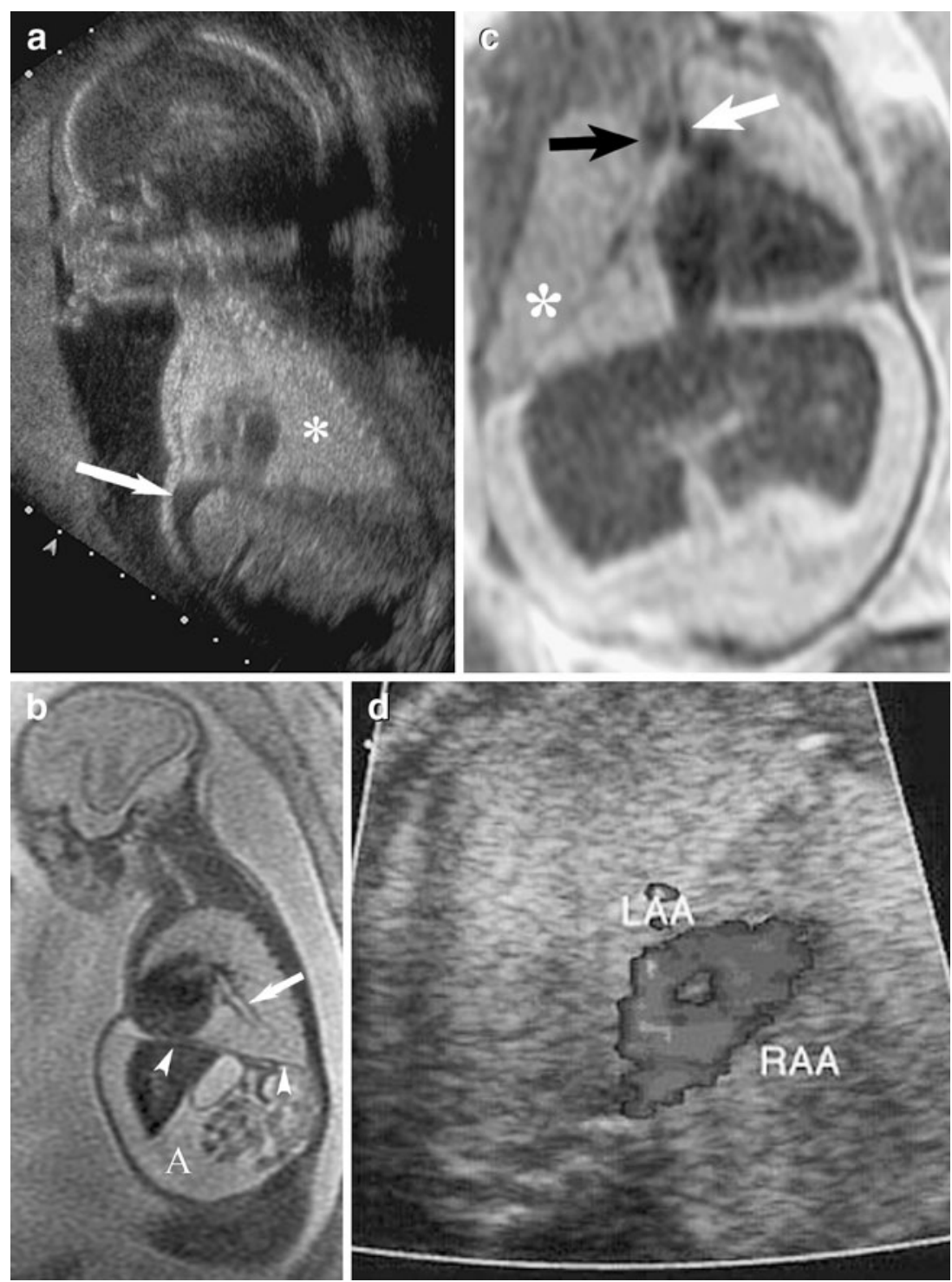

produce images that are easily understood by clinicians. Prenatal MRI can be used to delineate tumor extent, evaluate facial involvement and demonstrate the relationship of the mass to the trachea. Similarly, prenatal MRI can be used to confirm the diagnosis of CHAOS prior to undertaking fetal surgery [4].

\section{Conclusion}

The accurate prenatal diagnosis of upper airway obstruction is essential in order to provide appropriate management of this condition. The use of fetal MRI allows exquisite definition of fetal anatomy and facilitates treatment planning. The EXIT procedure has also demonstrated success in the treatment of intrinsic and extrinsic airway obstruction.
Acknowledgement Zhen J. Wang is supported by NIBIB T32 Training Grant 1 T32 EB001631.

Open Access This article is distributed under the terms of the Creative Commons Attribution Noncommercial License which permits any noncommercial use, distribution, and reproduction in any medium, provided the original author(s) and source are credited.

\section{References}

1. Woodward PJ, Sohaey R, Kennedy A et al (2005) From the archives of the AFIP: a comprehensive review of fetal tumors with pathologic correlation. Radiographics 24:215-242

2. Shum D, Coakley FV, Joe B et al (2007) Prenatal tracheal obstruction due to double aortic arch: a potential mimic of congenital high airway obstruction syndrome. AJR 188:W82-W85

3. Yedururi S, Guillerman RP, Chung T et al (2008) Multimodality imaging of tracheobronchial disorders in children. Radiographics 28:e29 
4. Coakley FV (2001) Role of magnetic resonance imaging in fetal surgery. Top Magn Reson Imaging 12:39-51

5. Coakley FV, Glenn OA, Qayyum A et al (2004) Fetal MRI: a developing technique for the developing patient. AJR 182:243-252

6. Coakley FV, Hricak H, Filly RA et al (1999) Complex fetal disorders: effect of MR imaging on management-preliminary clinical experience. Radiology 213:691-696

7. Mong A, Johnson AM, Kramer SS et al (2008) Congenital high airway obstruction syndrome: MR/US findings, effect on management, and outcome. Pediatr Radiol 38:1171-1179

8. Kuwashima S, Kitajima K, Kaji Y et al (2008) MR imaging appearance of laryngeal atresia (congenital high airway obstruc- tion syndrome): unique course in a fetus. Pediatr Radiol 38:344347

9. Guimaraes CV, Linam LE, Kline-Fath BM et al (2009) Prenatal MRI findings in congenital high airway sequence (CHAOS). Korean J Radiol 10:129-134

10. Hirose S, Farmer DL, Lee $\mathrm{H}$ et al (2004) The ex utero intrapartum treatment procedure: looking back at the EXIT. J Pediatr Surg $39: 375-380$

11. Wagner W, Harrison MR (2002) Fetal operations in the head and neck area: current state. Head Neck 24:482-490

12. Bouchard S, Johnson MP, Flake AW et al (2002) The EXIT procedure: experience and outcome in 31 cases. J Pediatr Surg 37:418-426 